\title{
Dynamic Zero Finding for Algebraic Equations
}

\author{
T. Mylvaganam, R. Ortega, J. Machado and A. Astolfi
}

\begin{abstract}
In a variety of contexts, for example the solution of differential games and the control of power systems, the design of feedback control laws requires the solution of nonlinear algebraic equations: obtaining such solutions is often not trivial. Motivated by such situations we consider systems of nonlinear algebraic equations and propose a method for obtaining their solutions. In particular, a dynamical system is introduced and (locally) stabilizing control laws which ensure that elements of the state converge to a solution of the algebraic equations are given. Illustrative numerical examples are provided. In addition it is shown that the proposed method is applicable to determine the equilibria of electrical networks with constant power loads.
\end{abstract}

\section{INTRODUCTION}

Often the design of feedback control laws involve obtaining solutions of nonlinear algebraic equations. This is, for example, the case when it comes to linear quadratic differential games and in many aspects of power systems analysis and design. In the former, when considering linear feedback strategies, the solution of a game requires solving a system of coupled Algebraic Riccati Equations (AREs) as seen, for instance, in [1]-[4]. In the latter, equilibria of a power system typically satisfy algebraic equations, for which the existence of solutions needs to be guaranteed (see, for example, [5]) and identified as part of the control design (see, for instance, [6]-[8]). With these motivations in mind, the focus of this paper is on zero finding for algebraic equations.

Similarities between classical problems in control theory and numerical analysis (in particular iterative numerical methods) have been exploited both in terms of analysis and design of iterative algorithms for solving algebraic equations (see, for example, [9]-[14]). Other approaches for zero finding using a control theoretic approach appear in [15], [16], where coupled AREs arising in the differential game formulation of mixed

This work has been partially supported by the European Union's Horizon 2020 Research and Innovation Programme under grant agreement No 739551 (KIOS CoE).

T. Mylvaganam is with the Department of Aeronautics, Imperial College London, London SW7 2AZ, UK, Email: t.mylvaganam@imperial.ac.uk.

R. Ortega and J. Machado are with the Laboratoire des Signaux et Systèmes, Ècole Supèrieure d'Electricitè (SUPELEC), Gif-sur-Yvette 91192, France, Email: ortega@lss.supelec.fr, juan.machado@12s.centralesupelec.fr.

A. Astolfi is with the Department of Electrical and Electronic Engineering, Imperial College London, London SW7 2AZ, UK and the DICII, Università di Roma "Tor Vergata", Via del Politecnico 1, 00133 Roma, Italy, Email: a.astolfi@imperial.ac.uk.
$H_{2} / H_{\infty}$ control problems have been considered. General algebraic equations have been considered in [17].

In this paper we consider general nonlinear algebraic equations for which we seek a solution. To this end a dynamical system, which is such that at any of its equilibria components of the state coincide with a solution of the algebraic equations, is introduced. Feedback control laws which, subject to certain conditions, locally asymptotically stabilize an equilibrium of the system are provided. The solution of the underlying algebraic equation can then be determined from the steady-state values of the trajectories of the closed-loop system. The main results of this paper can be summarised as follows. The problem of solving a system of algebraic equations is posed as a feedback stabilization problem for a dynamical system. A systematic method of constructing (locally) asymptotically stabilizing control laws is then proposed. The latter contribution draws inspiration from [18]. Therein the authors provide a method for constructing stabilizing control laws for systems with a particular structure with applications to power systems (see also [6], [19]). More precisely, the authors consider dynamical systems with a triangular structure and provide stabilizing feedback control laws through the introduction of an augmented system. The results presented in this paper are applicable to a wider class of problems.

The remainder of this paper is organised as follows. The problem considered is introduced in Section II. The main result, that is the design of a control law rendering the equilibria of a closed-loop system (locally) convergent to a solution of this problem, is provided in Section III. Remarks and numerical examples concerning some particular classes of algebraic equations are then provided in Sections IV and V. The results are then illustrated on a power systems problem in Section VI before some concluding remarks are given in Section VII.

Notation: The sets of natural numbers, real numbers and complex numbers are denoted by $\mathbb{Z}, \mathbb{R}$ and $\mathbb{C}$, respectively. The empty set is denoted by $\emptyset$. $I_{n}$ denotes the $n \times n$ identity matrix. Given $x \in \mathbb{R}^{n}$ we define $|x|^{2}=$ $x^{T} x$, and for a matrix $A, A^{\top}$ denotes its transpose, $\operatorname{vec}(A)$ denotes its vectorization and $\operatorname{adj}(A)$ denotes its adjoint matrix. Given a square matrix $A, \sigma(A)$ denotes its spectrum. The Kronecker product between two matrices $A$ and $B$ is denoted by $A \otimes B$. All functions are assumed sufficiently smooth. The gradient of a function $f$ is denoted by $\frac{\partial f}{\partial x}$. The Euclidean basis vectors are 
denoted by $e_{i} \in \mathbb{R}^{n}, i=1, \ldots, n$. The relations

$$
\begin{gathered}
\operatorname{vec}(A B C)=\left(C^{\top} \otimes A\right) \operatorname{vec}(B), \\
\operatorname{tr}(A C)=\operatorname{vec}\left(A^{\top}\right)^{\top} \operatorname{vec}(C),
\end{gathered}
$$

where $A, B$, and $C$ denote matrices of appropriate dimensions, are used repeatedly.

\section{PROBlem Formulation}

Let $f: \mathbb{R}^{n} \rightarrow \mathbb{R}^{n}$ denote a function described by ${ }^{1}$

$$
f(z)=q-P(z) z,
$$

where $z \in \mathbb{R}^{n}, q \in \mathbb{R}^{n}$ and $P: \mathbb{R}^{n} \rightarrow \mathbb{R}^{n \times n}$. Assume there exists $z^{*} \in \mathbb{R}^{n}$ such that

$$
q-P\left(z^{*}\right) z^{*}=0,
$$

and

$$
\operatorname{det}\left[P\left(z^{*}\right)\right] \neq 0
$$

Suppose, furthermore, that the image of $f$ around $z^{*}$ is onto a neighborhood of 0 . Note that (3) implies that $z^{*}$ is an isolated solution of (2).

Define the dynamical system

$$
\begin{aligned}
\dot{x} & =q-P(z) z, \\
\dot{z} & =u, \\
\dot{\Theta} & =W,
\end{aligned}
$$

where $x(t) \in \mathbb{R}^{n}, z(t) \in \mathbb{R}^{n}$ and $\Theta(t) \in \mathbb{R}^{n \times n}$ are the states of the system and $u(t) \in \mathbb{R}^{n}$ and $W(t) \in \mathbb{R}^{n \times n}$ denote control inputs. For this system we consider the problem of finding a static state feedback

$$
u=\hat{u}(x, z, \Theta), \quad W=\hat{W}(x, z, \Theta),
$$

such that the closed-loop system has an asymptotically stable equilibrium at $\left(0, z^{*}, P\left(z^{*}\right)\right)$. Thus, the second component of the steady-state of any trajectories converging to this equilibrium provide a solution to the algebraic equations $f(z)=0$.

Remark 1: The assumption that the image of $f$ is onto a neighbourhood of 0 around $z^{*}$ expresses nothing else than Brockett's necessary condition for the feedback stabilization, via smooth feedback, of system (4), see [20].

\section{MAIN RESULT}

\section{A. Design of the control law}

To provide a solution to the aforementioned problem we introduce a variable $z_{d} \in \mathbb{R}^{n}$, which is implicitly defined by the relation

$$
q-\Theta z_{d}=-\phi(x),
$$

where $\phi: \mathbb{R}^{n} \rightarrow \mathbb{R}^{n}$ is selected such that

$$
\begin{aligned}
\phi(0) & =0, \\
x^{\top} \phi(x) & >0, \forall x \neq 0 .
\end{aligned}
$$

\footnotetext{
${ }^{1}$ We recall that any differentiable function $f$ can be written in the form (1), but that the selection of $P$ may be non-unique.
}

For instance one could select $\phi(x)=\epsilon \tanh (x)$, for $\epsilon>0$, or $\phi(x)=x$. Note that, provided $\Theta(t)$ is invertible for all $t \geq 0$,

$$
z_{d}=\hat{z}_{d}(x, \Theta):=\Theta^{-1}[q+\phi(x)] .
$$

The main contribution of this paper, provided in Section III-C, is the construction of a feedback control law which locally asymptotically stabilizes an equilibrium of the system (4)-(5). A Lyapunov-like design approach is adopted to construct the control law. To this end, define the state vector $X:=\operatorname{col}(x, z, \operatorname{vec}(\Theta)) \in \mathbb{R}^{2 n+n^{2}}$ and consider the function

$$
V(X)=V_{1}(x)+V_{2}(X)+V_{3}(z, \operatorname{vec}(\Theta)),
$$

where ${ }^{2}$

$$
\begin{aligned}
& V_{1}(x)=\frac{1}{2}|x|^{2}, \quad V_{2}(X)=\frac{1}{2}\left|z-z_{d}\right|^{2}, \\
& V_{3}(z, \operatorname{vec}(\Theta))=\frac{1}{2} \operatorname{tr}\left((\Theta-P)^{\top}(\Theta-P)\right),
\end{aligned}
$$

Noting that $\operatorname{tr}\left((\Theta-P)^{\top}(\Theta-P)\right)=\sum_{i, j}\left(\theta_{i j}-p_{i j}\right)^{2}$, it follows that $V(X)>0$ for all $X \neq\left(0, z_{d}, \operatorname{vec}(P)\right)$ and $V(X)=0$ for $X=\left(0, z_{d}, \operatorname{vec}(P)\right)$.

The time derivatives of each of the component functions of $V$ along the trajectories of the system (4)-(6) are given by

$$
\begin{aligned}
\dot{V}_{1}= & -x^{\top} \phi-x^{\top} P\left(z-z_{d}\right)+\left(z_{d}^{\top} \otimes x^{\top}\right) \operatorname{vec}(\Theta-P), \\
\dot{V}_{2}= & \left(z-z_{d}\right)^{\top} u-\left(z-z_{d}\right)^{\top} \Theta^{-1} \dot{\phi} \\
& \quad+\left(z-z_{d}\right)^{\top} R(x, \Theta) \operatorname{vec}(W),
\end{aligned}
$$$$
\dot{V}_{3}=\operatorname{vec}(\Theta-P(z))^{\top} \operatorname{vec}(W)-\operatorname{vec}(\Theta-P) P_{z}(z) u,
$$

where we have defined the $n \times n^{2}$ matrix

$$
R(x, \Theta):=\left((q+\phi)^{\top} \Theta^{-\top}\right) \otimes \Theta^{-1},
$$

and the $n^{2} \times n$ matrix

$$
P_{z}(z):=\left[\begin{array}{lll}
\operatorname{vec}\left(\frac{\partial P}{\partial z_{1}}\right) & \ldots & \operatorname{vec}\left(\frac{\partial P}{\partial z_{n}}\right)
\end{array}\right],
$$

and used the fact that $\dot{z}_{d}=\Theta^{-1} \dot{\phi}-\Theta^{-1} \dot{\Theta} \Theta^{-1}(q+\phi(x))$. It follows that

$$
\begin{aligned}
\dot{V}= & -x^{\top} \phi \\
& +\left(z-z_{d}\right)^{\top}\left[u+\operatorname{Rvec}(W)-P^{\top} x-\Theta^{-1} \dot{\phi}\right] \\
& +\operatorname{vec}(\Theta-P)^{\top}\left[-P_{z} u+\operatorname{vec}(W)+\left(z_{d}^{\top} \otimes x^{\top}\right)^{\top}\right] .
\end{aligned}
$$

Now, define the matrix $M \in \mathbb{R}^{\left(n+n^{2}\right) \times\left(n+n^{2}\right)}$ as

$$
M(X):=\left[\begin{array}{cc}
I_{n} & R(x, \Theta) \\
-P_{z}(z) & I_{n^{2}}
\end{array}\right] .
$$

It is clear from (10) that selecting the control $u$ and $\operatorname{vec}(W)$ to satisfy

$$
\begin{aligned}
M(X) & {\left[\begin{array}{c}
u \\
\operatorname{vec}(W)
\end{array}\right] } \\
& =\left[\begin{array}{c}
-k_{z}\left(z-z_{d}\right)+P^{\top} x+\Theta^{-1} \dot{\phi} \\
-k_{\Theta} \operatorname{vec}(\Theta-P)-\left(z_{d}^{\top} \otimes x^{\top}\right)^{\top}
\end{array}\right],
\end{aligned}
$$

\footnotetext{
${ }^{2}$ To simplify the notation, when clear from the context, we omit
} the arguments of all mappings. 
where $k_{z}>0$ and $k_{\Theta}>0$ are tuning parameters, yields

$$
\dot{V}=-x^{\top} \phi-k_{z}\left|z-z_{d}\right|^{2}-k_{\Theta} \operatorname{tr}\left((\Theta-P)^{\top}(\Theta-P)\right) .
$$

Note that if we select $\phi(x)$ such that $x^{\top} \phi(x) \geq k_{x}|x|^{2}$, with $k_{x}>0$, we have $\dot{V} \leq-\min \left\{k_{x}, k_{z}, k_{\Theta}\right\} V$.

It is clear that the derivations above rely on the assumptions that $\Theta$ and $M(X)$ are invertible matricesan issue that is addressed in the following two subsections.

Remark 2: The assumption that $\Theta$ is invertible for all $t \geq 0$ can be interpreted as an observability condition on the output $z_{d}$ implicitly defined in (5).

\section{B. A preliminary lemma}

In this subsection we give a verifiable condition for the invertibility of $M(X)$. Towards this end, define the desired closed-loop equilibrium

$$
X_{e}:=\operatorname{col}\left(0, z^{*}, \operatorname{vec}\left(P\left(z^{*}\right)\right)\right) \in \mathbb{R}^{2 n+n^{2}},
$$

where $z^{*} \in \mathbb{R}^{n}$ is a solution of (2), the sets

$$
\begin{aligned}
\Omega_{\mu} & :=\left\{X \in \mathbb{R}^{2 n+n^{2}}|| X-X_{e} \mid \leq \mu\right\} \\
\mathcal{N} & :=\left\{X \in \mathbb{R}^{2 n+n^{2}} \mid \operatorname{det}(\Theta) \neq 0\right\}
\end{aligned}
$$

and the mapping $\Delta_{\delta}: \mathcal{N} \rightarrow \mathbb{R}^{n \times n}$

$$
\begin{gathered}
\Delta_{\delta}(X):=I_{n}+\frac{1}{(\operatorname{det}(\Theta))^{2}}\left[e_{1}^{\top} \operatorname{adj}(\Theta)(q+\delta) \operatorname{adj}(\Theta)\right. \\
\left.\ldots e_{n}^{\top} \operatorname{adj}(\Theta)(q+\delta) \operatorname{adj}(\Theta)\right] P_{z}(z),
\end{gathered}
$$

where $\delta \in \mathbb{R}^{n}$ is a parameter.

The following assumption is made to ensure the (local) existence of $u$ and $W$ satisfying (11).

Assumption 1: There exists $\epsilon>0$ and $\mu>0$ such that

$$
\operatorname{det}\left[\Delta_{\delta}(X)\right] \neq 0, \forall X \in \Omega_{\mu}, \forall|\delta| \leq \epsilon .
$$

Assumption 1 and the following lemma enables the design of the locally asymptotically stabilizing control laws provided in Section III-C (and in Section IV and Section $\mathrm{V}$ for linear and quadratic problems, respectively).

Lemma 1: Assumption 1 implies that $\operatorname{det}[M(X)] \neq 0$ for all $X \in \Omega_{\mu}$.

\section{A stabilizing controller}

To begin with we make the important observation that, in view of condition (3), the equilibrium $X_{e}$ is in the set $\mathcal{N}$. This allows to design a controller which locally stabilizes the equilibrium $X_{e}$ as established in the following proposition in which

$$
\mathcal{V}_{k}:=\left\{X \in \mathbb{R}^{2 n+n^{2}} \mid V(X) \leq k\right\},
$$

denotes the level sets of the function $V(X)$ defined in (8), with $z_{d}$ given in (7), and $\bar{k}=\max \left\{k: \mathcal{V}_{k} \subset \Omega_{\mu}\right\}$.
Thus, $\mathcal{V}_{\bar{k}}$ denotes the largest level set of $V$ contained in the set $\Omega_{\mu}$.

Proposition 1: Consider the dynamical system (4)-(5) and a function $\phi(x)$ satisfying (6). Suppose the condition (3) and Assumption 1 hold and let $X(0) \in \mathcal{V}_{\bar{k}}$. Then the dynamical system (4)-(5) in closed-loop with the control law $u$ and $W$ satisfying (11) is such that the equilibrium $X_{e}$ given in (13) is locally asymptotically stable and, in particular, the following implication is true

$$
\left[X(0) \in \Omega_{\mu} \cap \mathcal{N} \Rightarrow \lim _{t \rightarrow \infty} z(t)=z^{*}\right] .
$$

Remark 3: In [18] a method for designing stabilizing controllers for a class of triangular systems has been proposed. The method therein considers systems similar to (4) in which $P(z)$ is diagonal. The result provided in Proposition 1 is applicable to such systems, as well as a wider class of nonlinear systems.

Two special cases, namely systems of linear equations and systems of quadratic equations are considered in the following sections. Whereas in the former case $P(z)$ is a constant matrix, in the latter $P(z)$ is linear in $z$. Numerical examples are provided for both cases, which provide useful insights into the results expressed by Proposition 1.

\section{SySTEMS OF LINEAR EQUATIONS}

Consider the case in which $f$ defined in (1) is linear. In this case, $P(z)=\bar{P}$ is a constant matrix that, in view of (3), is full rank. We seek to obtain a solution of the system of linear equations

$$
q-\bar{P} z^{*}=0 \text {. }
$$

Recall the notation introduced previously, namely $X=$ $\operatorname{col}(x, z, \operatorname{vec}(\Theta))$ and $X_{e}=\operatorname{col}\left(0, z^{*}, \operatorname{vec}\left(P\left(z^{*}\right)\right)\right)$.

Corollary 1: Consider the system (4)-(5) with $P(z)=$ $\bar{P}$ and a function $\phi(x)$ satisfying (6). Let $\Theta(0)$ be such that

$$
\operatorname{det}(\Theta(0)) \operatorname{det}(\bar{P})>0 \text {. }
$$

Then there exists $u$ and $W$ satisfying (11) and the system (4)-(5) in closed-loop with $u$ and $W$ is such that $X_{e}$ is an asymptotically stable equilibrium and $\lim _{t \rightarrow \infty} z(t)=z^{*}$.

Remark 4: Corollary 1 establishes that for the case in which $P(z)$ is a constant matrix, the Assumption 1 in Proposition 1 is trivially satisfied provided $\operatorname{det}(\Theta(0))$ and $\operatorname{det}\left(P\left(z^{*}\right)\right)$ have the same sign, see (18).

Remark 5: In Proposition 1 it is assumed that $\bar{P}$ is full rank, such that (17) has a unique solution. If instead $\bar{P} \in \mathbb{R}^{m \times n}$, with $m<n$, (17) is an underdetermined system and may have no solutions or infinitely many solutions. In the latter case Corollary 1 may be generalised to achieve local asymptotic stability for an equilibrium corresponding to a solution of the underdetermined system. 


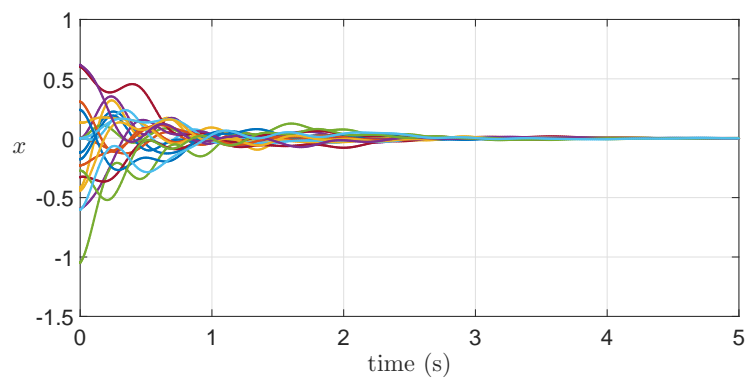

Fig. 1. Time histories of the component of $x$.
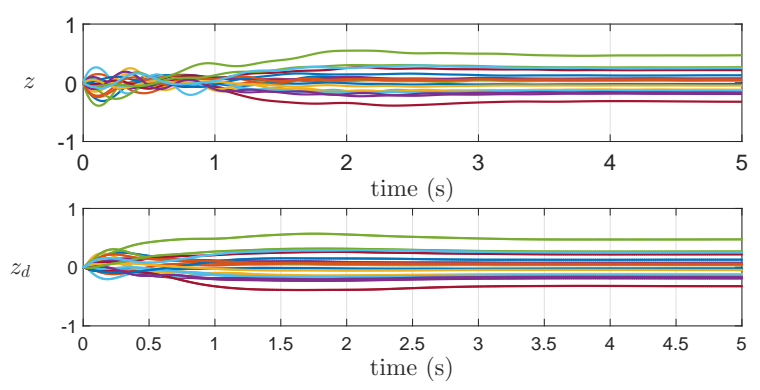

Fig. 2. Time histories of the components of $z$ (top) and $z_{d}$ (bottom).

\section{A. A numerical example}

A system of linear equations, as studied in Section IV, is considered and Corollary 1 is used to obtain the solution of (17).

Considering the case in which $n=20$, the parameters $\bar{P}$ and $q$ are randomly generated in MATLAB ${ }^{3}$ such that $\bar{P}$ is full rank. The system (4)-(5) in closed-loop with the feedback control law satisfying (11) is simulated with $^{4} \phi(x)=x, k_{z}=1$ and $k_{\Theta}=1$ and with the initial condition $z(0)=0, z_{d}(0)=0, \Theta(0)=I_{n}$ and $x(0)=q$. Note that the initial condition is such that $\operatorname{det}(\Theta(0)) \operatorname{det}(\bar{P})>0$ as required in (18) and Corollary 1 . The time histories of the components of $x$ are shown in Figure 1 and the time histories of the components of $z$ (top) and $z_{d}$ (bottom) are shown in Figure 2.

\section{SYSTEMS OF QUADRATIC EQUATIONS}

Consider now the case in which (1) is quadratic, i.e. the case in which $P(z)$ defined in (9) is linear in $z$. In this case $P_{z}(z)=\bar{P}_{z}$ is a constant matrix.

Let

$$
\begin{gathered}
\bar{\Delta}_{\delta}=I_{n}+\frac{1}{\left(\operatorname{det}\left(P\left(z^{*}\right)\right)\right)^{2}}\left[e_{1}^{\top} \operatorname{adj}\left(P\left(z^{*}\right)\right)(q+\delta) \operatorname{adj}\left(P\left(z^{*}\right)\right)\right. \\
\left.\ldots e_{n}^{\top} \operatorname{adj}\left(P\left(z^{*}\right)\right)(q+\delta) \operatorname{adj}\left(P\left(z^{*}\right)\right)\right] \bar{P}_{z},
\end{gathered}
$$

\footnotetext{
${ }^{3}$ The system of equations was randomly generated in MATLAB and can be replicated using the MATLAB code "n=20; rng('default'); SYS = rss(n); P = -(SYS.A - eye(n)); q = SYS.B;"

${ }^{4}$ The selection of the function $\phi$ has been made in accordance with (6).
}

TABLE I

THE SELECTION OF INITIAL CONDITIONS CONSIDERED IN THE SIMULATIONS.

\begin{tabular}{c||c|c} 
& $\Theta(0)$ & $z_{d}(0)$ \\
\hline \hline $\mathcal{I}_{4}$ & $\Theta_{0,1}$ & $z_{d 0,4}$ \\
$\mathcal{I}_{3}$ & $\Theta_{0,1}$ & $z_{d 0,3}$ \\
$\mathcal{I}_{2}$ & $\Theta_{0,1}$ & $z_{d 0,2}$ \\
$\mathcal{I}_{-4}$ & $\Theta_{0,2}$ & $z_{d 0,-4}$ \\
$\mathcal{I}_{-3}$ & $\Theta_{0,2}$ & $z_{d 0,-3}$ \\
$\mathcal{I}_{-2}$ & $\Theta_{0,2}$ & $z_{d 0,-3}$
\end{tabular}

Corollary 2: Consider the case in which (1) is a system of quadratic equations. Let $z^{*}$ denote a solution of (1) such that the condition (3) is satisfied. Suppose

$$
\sigma\left(\bar{\Delta}_{\delta}\right) \cap 0=\emptyset,
$$

for all $|\delta|<\epsilon$, and for some $\epsilon>0$. It follows that Assumption 1 is satisfied.

Remark 6: For the case in which (1) is quadratic, since $P_{z}$ is constant, Assumption 1 is a condition on $\Theta(0)$ (and $P(z *)$ ).

\section{A. A numerical example}

Consider the quadratic equation

$$
f(z)=\underbrace{\left[\begin{array}{c}
q_{1} \\
q_{2}
\end{array}\right]}_{q}-\underbrace{\left[\begin{array}{c}
-z_{1} z_{2} \\
z_{1}^{2}+z_{2}^{2}
\end{array}\right]}_{P(z) z}=0,
$$

where $q_{1} \in \mathbb{R}$ and $q_{2} \in \mathbb{R}$. Consider the selection $P(z)=$ $\left[\begin{array}{cc}-z_{2} & 0 \\ z_{1} & z_{2}\end{array}\right]$, and consider the case in which $q_{1}=8$ and $q_{2}=16$. In this case the system of equations (20) has two solutions.

Following the main result given in Proposition 1, the system (4)-(5) in closed-loop with the feedback given by (11) is simulated for different selections of parameters. Let $\Theta_{0,1}=\operatorname{diag}\{-1,1\}$ and $\Theta_{0,2}=\operatorname{diag}\{1,-1\}$ and let $z_{d 0, i}=[0, i]$, for $i \in \mathbb{Z}$. The initial condition $\Theta(0)$ is varied between $\Theta_{0,1}$ and $\Theta_{0,2}$, whereas $z_{d}(0)=z_{d 0, i}$, for $i=-4, \ldots, 4$. The considered initial conditions are referred to according to Table I. The remainder of the parameters are selected as follows ${ }^{5}: \phi=x, z(0)=0$, $x(0)=-q-\Theta(0) z_{d}(0)$ and $k_{z}=k_{\Theta}=200$.

The trajectories of $z$ are shown in Figures 3 for the initial conditions $\mathcal{I}_{4}$ (solid, black line), $\mathcal{I}_{3}$ (solid, dark grey line), $\mathcal{I}_{2}$ (solid, light gray line), $\mathcal{I}_{-4}$ (dashed, black line), $\mathcal{I}_{-3}$ (dashed, dark grey line) and $\mathcal{I}_{-2}$ (dashed, light grey line). The solid, square markers denote the initial conditions, whereas the solutions of (2) to which the trajectories converge are denoted by the diamondshaped markers.

\footnotetext{
${ }^{5}$ As in Section IV-A, the selection of the function $\phi$ has been made in accordance with (6).
} 


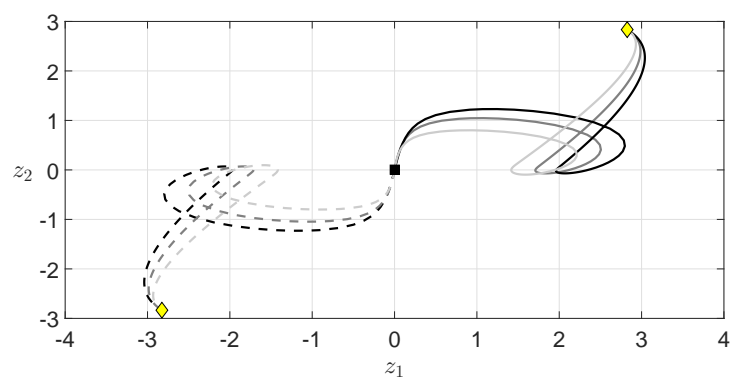

Fig. 3. Trajectories of $z$ for the initial conditions $\mathcal{I}_{4}$ (solid, black line), $\mathcal{I}_{3}$ (solid, dark grey line), $\mathcal{I}_{2}$ (solid, light gray line), $\mathcal{I}_{-4}$ (dashed, black line), $\mathcal{I}_{-3}$ (dashed, dark grey line) and $\mathcal{I}_{-2}$ (dashed, light grey line).
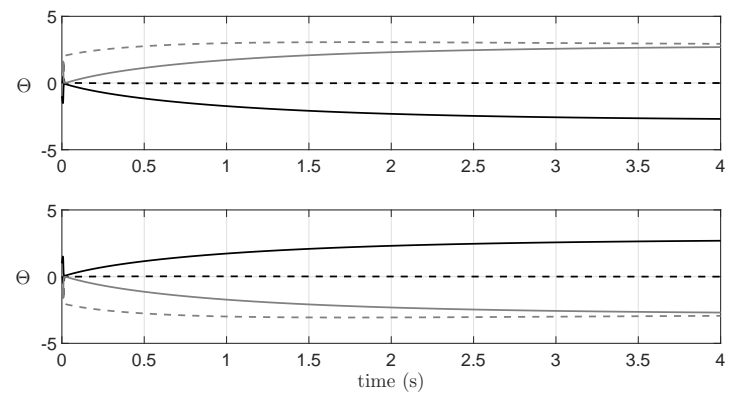

Fig. 4. Time histories of each component of $\Theta$ corresponding to the intitial conditions $\mathcal{I}_{4}$ (top) and $\mathcal{I}_{-4}$ (bottom).

Let $\Theta=\left[\begin{array}{ll}\Theta_{11} & \Theta_{12} \\ \Theta_{21} & \Theta_{22}\end{array}\right]$. The time histories of $\Theta_{11}$ (solid, black lines), $\Theta_{12}$ (dashed, black lines), $\Theta_{21}$ (dashed, grey lines) and $\Theta_{22}$ (solid, grey lines) are shown in Figure 4 for $\mathcal{I}_{4}$ (top) and $\mathcal{I}_{-4}$ (bottom).

\section{Vi. Application to Electrical Networks With CONSTANT POWER LOADS}

In this section we apply the developed numerical method to compute the steady state of an $m$-port linear time-invariant AC electrical network with Constant Power Loads (CPLs) working in sinusoidal steady-state at a frequency $\omega_{0} \in \mathbb{R}_{+}$, see [5] and references therein for additional details on this problem.

The description of this regime in the frequency domain is given by the relation

$$
\bar{V}\left(j \omega_{0}\right)=G\left(j \omega_{0}\right) I\left(j \omega_{0}\right)+K\left(j \omega_{0}\right),
$$

where $^{6} \bar{V} \in \mathbb{C}^{m}$ and $I \in \mathbb{C}^{m}$ are the vectors of generalized Fourier transforms of the port voltages and currents, respectively, and $K \in \mathbb{C}^{m}$ captures the effect of the external (current or voltage) AC sources, all evaluated at the frequency $\omega_{0}$.

Defining the apparent power at the CPLs as

$$
S_{i}:=\mathcal{P}_{i}+j \mathcal{Q}_{i}, \quad i \in\{1, \ldots m\},
$$

\footnotetext{
${ }^{6} \mathrm{To}$ simplify the notation the argument $j \omega_{0}$ is omitted in the sequel.
}

where $\mathcal{P}_{i} \in \mathbb{R}$ and $\mathcal{Q}_{i} \in \mathbb{R}$ are the active and reactive power at port $i$, respectively, the CPLs constraint the network via

$$
\bar{V}_{i} I_{i}^{\mathrm{c}}=-S_{i},
$$

where $(\cdot)^{c}$ denotes the complex conjugate operator.

Obviously, a necessary and sufficient condition for the existence of a sinusoidal steady-state (at a given frequency $\omega_{0}$ ) is that the complex equations (21), (22) and (23) have a solution. It has been shown in [5] that this set of equations can be written in the form

$$
q-P(z) z=0,
$$

where $q:=\operatorname{col}(\mathcal{P}, \mathcal{Q}), z:=\operatorname{col}(\operatorname{Re}\{I\}, \operatorname{Im}\{I\})$ and

$$
\begin{aligned}
& P(z)= \\
& -\left[\begin{array}{cc}
\operatorname{diag}\left\{e_{i}^{\top} \bar{G} z+\bar{K}_{i}\right\} & \operatorname{diag}\left\{e_{m+i}^{\top} \bar{G} z+\bar{K}_{m+i}\right\} \\
\operatorname{diag}\left\{e_{m+i}^{\top} \bar{G} z+\bar{K}_{m+i}\right\} & -\operatorname{diag}\left\{e_{i}^{\top} \bar{G} z+\bar{K}_{i}\right\}
\end{array}\right], \\
& \text { with } \bar{K}=\operatorname{col}(\operatorname{Re}\{K\}, \operatorname{Im}\{K\}) \text { and } \\
& \qquad \bar{G}=\left[\begin{array}{cc}
\operatorname{Re}\{G\} & -\operatorname{Im}\{G\} \\
\operatorname{Im}\{G\} & \operatorname{Re}\{G\}
\end{array}\right] .
\end{aligned}
$$

Hence, the problem of finding the equilibrium current $I$ can be solve using the method presented in this paper.

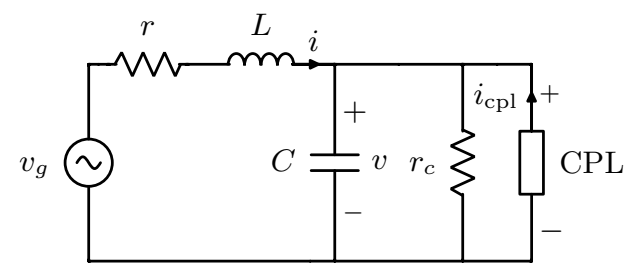

Fig. 5. AC Linear RLC circuit with a CPL.

Consider as an example the circuit shown in Fig. 5 with the voltage source given by

$$
v_{g}(t)=\sqrt{2} V_{g} \cos \left(\omega_{0} t\right)
$$

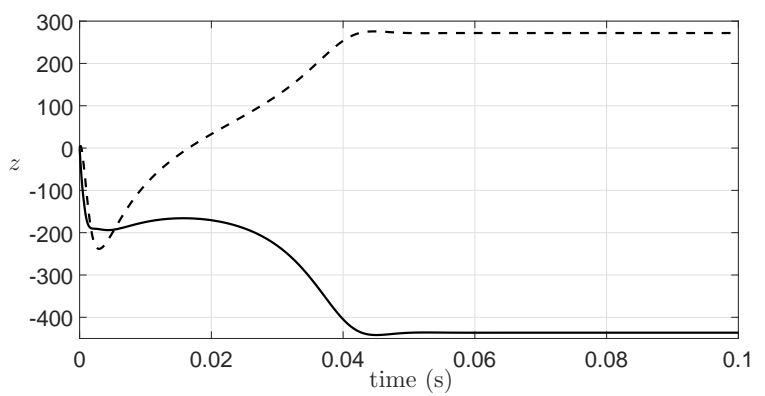

Fig. 6. Time histories of the first (solid line) and second (dashed line) components of $z$, which converge to the steady-state current corresponding to the network of Fig. 5. 
and the CPL extracting power from the network according to (22). In this case $G$ and $K$ are given by

$$
\begin{aligned}
& G=\frac{r+j L w_{0}}{\left(1+\frac{r}{r_{c}}-L C w_{0}^{2}\right)+j\left(r C+\frac{L}{r_{c}}\right) w_{0}} \\
& K=\frac{V_{g}}{\left(1+\frac{r}{r_{c}}-L C w_{0}^{2}\right)+j\left(r C+\frac{L}{r_{c}}\right) w_{0}} .
\end{aligned}
$$

Using the circuit parameters from Table VI, the above expressions result in $G=0.0412+j 0.0238, \quad K=$ $24.3592-j 0.6219$.

TABLE II

PARAMETERS FOR THE CIRCUIT IN FIG. 5

\begin{tabular}{c|c|c|c}
\hline \hline$r=0.04 \Omega$ & $L=78.0 \mu \mathrm{H}$ & $C=2.0 \mathrm{mF}$ & $V_{g}=24 \mathrm{~V}$ \\
\hline$r_{c}=5 \mathrm{k} \Omega$ & $\mathcal{P}=100 \mathrm{~W}$ & $\mathcal{Q}=-50 \mathrm{var}$ & $\omega_{0}=2 \pi 50 \mathrm{rad} / \mathrm{s}$ \\
\hline \hline
\end{tabular}

The existence of steady-states is guaranteed by [5, Proposition 3]. However, no indication is given on how to find these. In what follows, the main result of this paper, i.e. Proposition 1, is used to determine the steady-state current $I$ of the circuit shown in Figure 5 with the circuit parameters given in Table VI.

Consider the dynamical system (4)-(5) with $P(z)$ given in (24). The system (4)-(5) in closed-loop with the control law satisfying (11), with $\phi(x)=100 x$, is simulated with the following selection of control parameters and initial conditions: $k_{z}=10, k_{\Theta}=600$, $z(0)=[-1,0]^{\top}, \Theta(0)=10 I, z_{d}(0)=0$ and $x(0)=$ $-q-\Theta(0) z_{d}(0)$. The resulting time histories of the first (solid line) and second (dashed line) component of the state $z$ are shown in Figure 6. The trajectories converge to the solution $z^{*}=\operatorname{col}(\operatorname{Re}\{I\}, \operatorname{Im}\{I\})=$ $\operatorname{col}(-436.3895,271.827)$.

\section{CONCLUSION}

We consider the problem of obtaining solutions for general, nonlinear, algebraic equations through the introduction of a dynamical system. The dynamical system is constructed such that it has an equilibrium when elements of its state coincides with the solution of the algebraic equation. The problem is then solved through the design of a feedback control law which (locally) asymptotically stabilizes an equilibrium of the system. The proposed method is demonstrated on numerical examples and on the problem of determining the steady-state current of an AC power system.

Directions for future research include further analysis of the proposed method. In particular, the performance of the method for specific classes of problems including matrix equations, such as coupled AREs arising in the context of linear quadratic differential games, will be studied. When considering coupled AREs one often seeks a solution with certain properties (such as positive definiteness). It is therefore of interest to further extend the results presented in this paper to systematically ensure convergence to solutions with particular properties. It is also of interest to consider applications to problems arising in large-scale power systems control.

\section{REFERENCES}

[1] T. Basar and G. Olsder, Dynamic Noncooperative Game Theory. Academic Press, 1982.

[2] A. W. Starr and Y. C. Ho, "Nonzero-sum differential games," Journal of Optimization Theory and Applications, vol. 3, pp. 184206, 1969.

[3] - "Further properties of nonzero-sum differential games," Journal of Optimization Theory and Applications, vol. 3, pp. 207$219,1969$.

[4] T. Mylvaganam, M. Sassano, and A. Astolfi, "Constructive $\epsilon$-Nash equilibria for nonzero-sum differential games," IEEE Transactions on Automatic Control, vol. 60, no. 4, pp. 950-965, 2015.

[5] J. E. Machado, R. Griñó, N. Barabanov, R. Ortega, and B. Polyak, "On existence of equilibria of multi-port linear ac networks with constant-power loads," IEEE Transactions on Circuits and Systems I: Regular Papers, vol. 64, no. 10, pp. 2772-2782, 2017.

[6] D. Casagrande, A. Astolfi, D. Langarica, and R. Ortega, "Solution to the multi-machine transient stability problem and simulated validation in realistic scenarios," IET Generation, Transmission $\mathcal{E}$ Distribution, vol. 8, no. 8, pp. 1392-1405, 2014.

[7] J. Schiffer, E. Fridman, R. Ortega, and J. Raisch, "Stability of a class of delayed port-hamiltonian systems with application to microgrids with distributed rotational and electronic generation," Automatica, vol. 74, pp. 71-79, 2016.

[8] S. Trip, M. Bürger, and C. D. Persis, "An internal model approach to (optimal) frequency regulation in power grids with time-varying voltages," Automatica, vol. 64, pp. 240 - 253, 2016.

[9] A. Bhaya and E. Kaszkurewicz, "A control-theoretic approach to the design of zero finding numerical methods," IEEE Transactions on Automatic Control, vol. 52, no. 6, pp. 1014-1026, 2007.

[10] J. M. Ortega, "Stability of difference equations and convergence of iterative processes," SIAM Journal on Numerical Analysis, vol. 10, no. 2, pp. 268-282, 1973.

[11] K. Kashima and Y. Yamamoto, "System theory for numerical analysis," Automatica, vol. 43, no. 7, pp. 1156 - 1164, 2007.

[12] R. D. Harbor, "A control theory perspective of iterative numerical methods," in IEEE Proceedings of the 21st Southeastern Symposium on System Theory, 1989, pp. 71-73.

[13] T. Han and Y. Han, "Solving large scale nonlinear equations by a new ODE numerical integration method," in Applied Mathematics, vol. 1, no. 3, 2010, pp. 222-229.

[14] B. Polyak and P. Shcherbakov, "Lyapunov functions: An optimization theory perspective," in Proceedings of the 20th IFAC World Congress, vol. 50, no. 1, 2017, pp. 7456 - 7461.

[15] T. Mylvaganam and A. Astolfi, "A Nash game approach to mixed $H_{2} / H_{\infty}$ control for input-affine nonlinear systems," in Proceedings of the 10th IFAC Symposium on Nonlinear Control Systems, 2016.

[16] $ـ$,Dynamic algorithms for solving coupled algebraic riccati equations arising in mixed $H_{2} / H_{\infty}$ control for scalar linear systems," in Proceedings of the 55th IEEE Conference on Decision and Control (CDC), 2016, pp. 652-657.

[17] _ , "Zero finding via feedback stabilisation," in Proceedings of the 20th IFAC World Congress, vol. 50, no. 1, 2017, pp. 8133 8138.

[18] D. Casagrande, A. Astolfi, and R. Ortega, "Global stabilization of non-globally linearizable triangular systems: Application to transient stability of power systems," in Proceedings of the 50th IEEE Conference on Decision and Control and European Control Conference, 2011, pp. 331-336.

[19] D. Casagrande, A. Astolfi, R. Ortega, and D. Langarica, "A solution to the problem of transient stability of multimachine power systems," in Proceedings of the 51st IEEE Conference on Decision and Control (CDC), 2012, pp. 1703-1708.

[20] W. Wong and R. W. Brockett, "Systems with finite communication bandwidth constraints. I. state estimation problems," IEEE Transactions on Automatic Control, vol. 42, no. 9, pp. 1294-1299, 1997. 